\title{
Task Errors Drive Memories That Improve Sensorimotor Adaptation
}

\author{
Li-Ann Leow, ${ }^{1}$ Welber Marinovic, ${ }^{2}$ Aymar de Rugy, ${ }^{3}$ and $\mathbb{C}^{-T i m o t h y ~ J . ~ C a r r o l l ~}{ }^{1}$ \\ ${ }^{1}$ Centre for Sensorimotor Performance, School of Human Movement and Nutrition Sciences, University of Queensland, Brisbane, 4072 Queensland, \\ Australia, ${ }^{2}$ School of Psychology, Curtin University, Bentley, 6102 Western Australia, Australia, and ${ }^{3}$ Institut de Neurosciences Cognitives et Intégratives \\ d'Aquitaine, Centre National de la Recherche Scientifique Unité Mixte de Recherche 5287, Université de Bordeaux, France 33076
}

Traditional views of sensorimotor adaptation (i.e., adaptation of movements to perturbed sensory feedback) emphasize the role of automatic, implicit correction of sensory prediction errors. However, latent memories formed during sensorimotor adaptation, manifest as improved relearning (e.g., savings), have recently been attributed to strategic corrections of task errors (failures to achieve task goals). To dissociate contributions of task errors and sensory prediction errors to latent sensorimotor memories, we perturbed target locations to remove or enforce task errors during learning and/or test, with male/female human participants. Adaptation improved after learning in all conditions where participants were permitted to correct task errors, and did not improve whenever we prevented correction of task errors. Thus, previous correction of task errors was both necessary and sufficient to improve adaptation. In contrast, a history of sensory prediction errors was neither sufficient nor obligatory for improved adaptation. Limiting movement preparation time showed that the latent memories driven by learning to correct task errors take at least two forms: a time-consuming but flexible component, and a rapidly expressible, inflexible component. The results provide strong support for the idea that movement corrections driven by a failure to successfully achieve movement goals underpin motor memories that manifest as savings. Such persistent memories are not exclusively mediated by time-consuming strategic processes but also comprise a rapidly expressible but inflexible component. The distinct characteristics of these putative processes suggest dissociable underlying mechanisms, and imply that identification of the neural basis for adaptation and savings will require methods that allow such dissociations.

Key words: motor learning; motor memories; savings; sensorimotor adaptation; visuomotor rotation

\section{Significance Statement}

Latent motor memories formed during sensorimotor adaptation manifest as improved adaptation when sensorimotor perturbations are reencountered. Conflicting theories suggest that this "savings" is underpinned by different mechanisms, including a memory of successful actions, a memory of errors, or an aiming strategy to correct task errors. Here we show that learning to correct task errors is sufficient to show improved subsequent adaptation with respect to naive performance, even when tested in the absence of task errors. In contrast, a history of sensory prediction errors is neither sufficient nor obligatory for improved adaptation. Finally, we show that latent sensorimotor memories driven by task errors comprise at least two distinct components: a time-consuming, flexible component, and a rapidly expressible, inflexible component.

\section{Introduction}

Much of what we know about motor learning emerged from the paradigm of sensorimotor adaptation, where a systematic pertur-

Received June 27, 2019; revised Jan. 20, 2020; accepted Jan. 25, 2020.

Author contributions: L.-A.L. and T.J.C. designed research; L.-A.L. performed research; L.-A.L. analyzed data; L.-A.L. wrote the first draft of the paper; L.-A.L., W.M., A.d.R., and T.J.C. edited the paper; L.-A.L. and T.J.C. wrote the paper.

This work was supported by Australian Research Council Discovery Project Grant DP180103081, Australian Research Council Discovery Project Grant DP160102001, and the Brain Foundation Australia. We thank Aya Uchida, Tess Stevenson, and Tamara Spingler for assistance with data collection.

The authors declare no competing financial interests.

Correspondence should be addressed to Li-Ann Leow at l.leow@uq.edu.au. bation is applied to the visual representation of movement (von Helmholtz, 1924; Cunningham, 1989), or to limb dynamics (Dietz et al., 1994; Shadmehr and Mussa-Ivaldi, 1994). This changes the sensory consequences of motor commands, prompting adaptive motor responses to restore effective movement. Adaptation is essential to sustain movement success, due to ubiquitous changes within our bodies and the environment. However, the extent to which principles of sensorimotor adaptation apply to learning of motor skills remains unclear (Krakauer et al., 2019).

https://doi.org/10.1523/JNEUROSCI.1506-19.2020

Copyright $\odot 2020$ the authors 
A key piece of evidence addressing the question of how sensorimotor adaptation relates to other forms of motor learning is the extent to which adaptation generates long-lasting memories. Sensorimotor memory is often operationalized as improved adaptation when reencountering a similar perturbation. This phenomenon, commonly termed savings, implies latent memories (Ebbinghaus, 1964) because the benefit of previous learning persists for months even after behavior has returned to the naive state (Zarahn et al., 2008; Landi et al., 2011; Maeda et al., 2018). A latent form of sensorimotor memory is both obligatory for success given the nonstationarity of our environment, and reminiscent of learnt motor skills, which can be flexibly expressed according to context.

The mechanisms that underlie savings are unresolved. One idea suggests that savings occurs because specific actions become associated with success during learning, and these actions are recalled upon perturbation-induced failure (Huang et al., 2011). Another idea suggests a role of "meta-learning," where the structure of an experienced perturbation is encoded to assist subsequent adaptation (Braun et al., 2009). Yet another influential theory suggests that a systematic sequence of perturbation-induced errors generates a memory of errors, increasing sensitivity to those errors and the gain of error correction when reencountering similar errors (Gonzalez Castro et al., 2014; Herzfeld et al., 2014). In contrast, recent work provides strong evidence that savings is dominated by strategic selection of actions that restore success (Haith et al., 2015; Morehead et al., 2015; de Brouwer et al., 2017; Avraham et al., 2020). But is strategy use the sole contributor to latent sensorimotor memories, or is there some component of latent sensorimotor memories that are less accessible to conscious control? If there are indeed multiple components to sensorimotor memories, what are the necessary conditions for their encoding and expression? Sensorimotor perturbations, by definition, evoke sensory prediction errors (SPEs, or, discrepancies between predicted sensory outcomes of movements and actual sensory outcomes of movements). Perturbations also often evoke reward prediction errors (RPEs, or discrepancies between predicted and actual reward). In a goal-directed task, RPEs take the form of task error, or failure to achieve an internally determined task goal. Previous conceptualizations delineate the neural mechanisms of SPEs and RPEs, where the cerebellum uses SPEs to improve action execution, whereas the basal ganglia uses RPEs to improve action selection (Taylor and Ivry, 2014). However, recent evidence not only shows reciprocal connections between the two systems (Bostan and Strick, 2018), but also that the function of these systems is not completely dissociable (e.g., both SPEs and RPEs modulate cerebellar responses) (Heffley et al., 2018; Kostadinov et al., 2019). In sensorimotor learning, these errors can interact to influence adaptation (e.g., Pidoux et al., 2018; Leow et al., 2018; Kim et al., 2019). Could SPEs and RPEs jointly contribute to latent sensorimotor memories? To answer this question, we examined the contribution of task errors and SPEs to the encoding and expression of sensorimotor memories. We show that a history of adaptation to task errors, and not a history of adaptation to SPEs, is necessary to encode memories that subsequently improve adaptation. Further, we show that this memory consists of at least two components: a time-consuming but flexible component that readily adapts motor responses to counteract task errors in either direction (i.e., clockwise $[\mathrm{CW}]$ or counterclockwise [CCW] from the target), and a rapidly expressible, inflexible component that only counteracts task errors that are specific to the direction of errors encountered during initial adaptation, and actually worsens the adaptive responses to task error in the opposite direction.

\section{Materials and Methods}

Participants. There were a total of 144 participants ( 94 female, age range 17-34 years, mean age 20.5 years). All participants were naive to visuomotor rotation and force-field adaptation tasks, and were naive to the aims of the study. Participants received course credit or monetary reimbursement upon study completion. The study was approved by the $\mathrm{Hu}-$ man Research Ethics Committee at the University of Queensland. All participants provided written informed consent. This study conforms to the Declaration of Helsinki.

Apparatus. Participants completed the task using a vBOT planar robotic manipulandum, which has a low-mass, two-link carbon fiber arm and measures position with optical encoders sampled at $1000 \mathrm{~Hz}$ (Howard et al., 2009). Participants were seated on a height-adjustable chair at their ideal height for viewing the screen for the duration of the experiment. Visual feedback was presented on a horizontal plane on a 27 inch LCD computer monitor (ASUS, VG278H, set at $60 \mathrm{~Hz}$ refresh rate) mounted above the vBOT and projected to the participant via a mirror in a darkened room, preventing direct vision of her/his hand. The mirror allowed the visual feedback of the targets, the start circle, and hand cursor to be presented in the plane of movement, with a black background. The start was aligned $\sim 10 \mathrm{~cm}$ to the right of the participant's mid-sagittal plane at approximately mid-sternum level. An air-sled was used to support the weight of participants' right forearms, to reduce possible effects of fatigue.

General trial structure. While grasping the robot arm, participants moved their hand cursor $(0.5 \mathrm{~cm}$ radius red circle) from the central start circle $(0.5 \mathrm{~cm}$ radius white circle $)$ to the targets $(0.5 \mathrm{~cm}$ radius yellow circles). Targets appeared in random order at 1 of 8 locations $\left(0^{\circ}, 45^{\circ} \ldots\right.$ $315^{\circ}$ ) at a radius of $9 \mathrm{~cm}$ from a central start circle. At the start of each trial, the central start circle was displayed. If participants failed to move their hand cursor to within $1 \mathrm{~cm}$ of the start circle after $1 \mathrm{~s}$, the robotic manipulandum moved the participant's hand to the start circle (using a simulated $2 \mathrm{D}$ spring with the spring constant magnitude increasing linearly over time). A trial was initiated when the cursor remained within the home location at a speed $<0.1 \mathrm{~cm} / \mathrm{s}$ for $200 \mathrm{~ms}$. Across all experiments, we used a classical timed-response paradigm (e.g., Schouten and Bekker, 1967) to manipulate movement preparation time during the planar reaching task (Favilla and De Cecco, 1996). A sequence of three tones, spaced $500 \mathrm{~ms}$ apart, was presented at a clearly audible volume via external speakers. Participants were instructed to time the onset of their movements with the onset of the third tone, which was more highly pitched than the two previous tones, and then to slice through the target with their cursor. Movement initiation time was identified online as when hand speed exceeded $2 \mathrm{~cm} / \mathrm{s}$. Targets appeared at $1000 \mathrm{~ms}$ (Experiments 1-3) or $250 \mathrm{~ms}$ (Experiment 4) minus a monitor display latency $(27.6 \pm 1.8 \mathrm{~ms})$, before the third tone. Thus, target direction information became available $972 \mathrm{~ms}$ before the desired initiation time for Experiments 1-3 and $222 \mathrm{~ms}$ before the desired initiation time for Experiment 4. When movements were initiated $50 \mathrm{~ms}$ later than the third tone, the trial was aborted: the screen went black and the text "Too Late" was displayed on the feedback screen. When movements were initiated $>100$ ms before the desired initiation time, the trial was aborted: the screen went black and a "Too Soon" error message was displayed. Thus, movements had to be initiated between 872 and $1022 \mathrm{~ms}$ of target presentation in Experiments 1-3 and between 122 and $272 \mathrm{~ms}$ of target presentation in Experiment 4 . We chose this movement preparation time for consistency with our previous work using the timed-response paradigm with visuomotor rotations (Leow et al., 2017). No visual feedback about movements was available when trials were aborted, and so such trials were repeated at the end of the cycle. We enforced long movement preparation times across Experiments 1-3 to prevent the possibility that the task error manipulation resulted in self-selection of different movement preparation times. Under these conditions, participants had ample opportunity (i.e., time for movement preparation) to use explicit strategies.

Across all conditions, cursor feedback was displayed after the hand had moved $4 \mathrm{~cm}$ from the start to target (located $9 \mathrm{~cm}$ away from the start). At 
this point, the direction of cursor velocity was measured to define target movements in some conditions as described below. During StandardTaskError conditions, the target remained stationary throughout the trial, such that whether or not participants hit the target was contingent on the participant's reach direction (see Fig. 1A). During NoTaskError conditions, the target was shifted to align with the direction of cursor velocity, measured at $4 \mathrm{~cm}$ into the movement. This is analogous to moving a basketball hoop toward the ball mid-flight; the ball always goes through the hoop regardless of the person's actions. During RandomTaskError conditions, the target was shifted randomly by $20^{\circ}-30^{\circ}$ (CCW in half of the trials, $\mathrm{CW}$ in half of the trials) relative to the cursor direction when the hand had moved $4 \mathrm{~cm}$ from the start. This is analogous to moving a basketball hoop away from the ball's trajectory; participants can never get the ball through the hoop regardless of where they shoot. In Experiments 3 and 4, we imposed systematic task errors without any perturbation of the hand cursor relationship: the target was moved during the movement by $30^{\circ}$ relative to the original target position, always in the same direction (CW for half of all participants, CCW for the other half of participants, counterbalanced): no rotation of the visual feedback of movement was imposed when this occurred.

To familiarize participants with the equipment and the timedresponse paradigm, all participants were first allowed a familiarization block comprising a maximum of 6 cycles. One cycle consisted of 1 trial to each of the 8 targets, and target order was random within each cycle. Participants were explicitly instructed to make shooting movements so that the cursor sliced through the targets, rather than to stop on the targets. Cursor feedback terminated as soon as the desired movement extent (the $9 \mathrm{~cm}$ distance between the start and the target) was achieved. After familiarization, all participants (regardless of assigned condition) were given the same task instruction, as follows. "Your task in this experiment is to hit the targets. The computer might disturb the cursor and/or the target, this is a normal part of the experiment, just try to hit the target as well as you can." Participants then completed the following blocks. Baseline ( 6 cycles): no rotation of visual feedback. Training (60 cycles): For Experiments 1 and 2, a $30^{\circ}$ rotation of cursor feedback representing the hand position was imposed. Half of all participants encountered a $\mathrm{CW} 30^{\circ}$ cursor rotation and half encountered a $30^{\circ} \mathrm{CCW}$ cursor rotation. For Experiments 3 and 4, no cursor rotation was applied during training, but a $30^{\circ}$ rotation of target position relative to the original target position was applied mid-movement (see Figs. 3, 4). Half of all participants encountered a CW $30^{\circ}$ target rotation and half encountered a $30^{\circ} \mathrm{CCW}$ target rotation. No feedback ( 6 cycles): Upon leaving the start circle, no feedback about movements was available. Before this block, participants received explicit instructions about the rotation removal, as follows: "Any disturbance that the computer has applied is now gone, and the feedback of your movement will now be hidden as soon as it leaves the start circle, so please move straight to the target." Washout: Cursor position feedback was restored, but the $30^{\circ}$ rotation of cursor (or target) was removed. For Experiments 1 and 2, to prevent the experience of washoutrelated task errors, task errors were removed across all conditions (i.e., the target position shifted mid-movement to ensure that the cursor always hit the target). The length of the washout block was the same as the adaptation block (60 cycles). For Experiments 3 and 4, participants had no prior experience of the cursor rotation, only task errors, and they could volitionally reach straight to the target by the end of the nofeedback block; thus, it was unnecessary to use a long washout with the no-task-error manipulation to avoid exposure to errors related to abrupt removal of the perturbation: we thus provided 12 washout cycles without mid-movement target shifts. Test ( 60 cycles): the $30^{\circ}$ rotation of cursor feedback was imposed (half of all participants encountered a CW $30^{\circ}$ rotation and half encountered a $30^{\circ} \mathrm{CCW}$ rotation). Between each block, there was a small delay to allow for experimental instructions and loading of the computer code for different experimental blocks.

Data analysis. Movement onset time was taken as the time at which hand speed first exceeded $2 \mathrm{~cm} / \mathrm{s}$. Reach directions were quantified at $20 \%$ of the radial distance between the start and the target. Reaches with absolute initial direction errors $>60^{\circ}$ with respect to the target (movements that were $>60^{\circ}$ to the left or the right of the target) were considered outliers and were removed from analyses. Experiment 1:
StandardTaskErrors, $0.62 \%$; NoTaskErrors, $0.11 \%$; EnforcedTaskErrors, $1.73 \%$. Experiment 2: StandardTE ${ }^{\text {Train }}{ }^{N o T E}{ }^{\text {Test }}, 0.29 \%$; RandomTE ${ }^{\text {Train }}{ }^{\text {NoTE }}{ }^{\text {Test }}, 0.62 \%$; NoTE ${ }^{\text {Train }}$ StdTE $^{\text {TEST }}, 0.17 \%$. Experiment 3: Same, 0.30\%; Different, 0.20\%. Experiment 4: ShortDifferent, 3.82\%; ShortSame, 4.31\%; ShortNaive, $4.59 \%$. Excluding these trials did not have any qualitative impact on the results. Trials were averaged in cycles of 8 (one trial for each target angle). For graphing purposes, reach directions for participants who experienced CW rotations were signtransformed and pooled with data for participants who experienced CCW rotations: values closer to $30^{\circ}$ indicate more complete adaptation.

We estimated adaptation performance as percent adaptation, which quantifies reach directions in every cycle relative to the ideal reach direction (as shown by Hadjiosif and Smith, 2013), we estimated percent adaptation by calculating reach directions as a percentage of ideal reach directions resulting from perfect adaptation performance as follows:

$$
\text { percent adaptation }=100 \% \times \frac{\text { reach direction }}{\text { ideal reach direction }}
$$

It is important to consider how individual differences at baseline affect our measures of adaptation. Previous methods of accounting for this by subtracting premanipulation/intervention behavior from postmanipulation/intervention behavior is more sensitive to noisy baselines and risk of Type 2 error (Vickers, 2001, 2014). To account for preperturbation baseline biases, we entered percent adaptation averaged from the final 3 cycles before rotation onset (i.e., percent adaptation in the last 3 baseline cycles, or percent adaptation in the last 3 washout cycles) as covariates in all of our ANCOVAs. We chose this method as simulation studies show that this method maintains statistical power (1) when there is poor correlation between baseline and follow-up measures, (2) whether the change from baseline to follow-up is a shift in raw scores or as a ratio (e.g., treatment reduced scores by $\mathrm{x} \%$ ), and (3) whether the control group also changes from baseline to follow-up (Vickers, 2001, 2014). Importantly, analyses outcomes were similar regardless of whether we (1) did not account for individual differences at baseline, (2) subtracted preperturbation behavior from postperturbation behavior, or (3) when we accounted for baseline by entering them as covariates.

Before analysis, we first divided adaptation into early (cycles 1-20), middle (cycles 21-40), and late phase (cycles 41-60). To estimate improved (i.e., better-than-naive) adaptation, we compared naive with test reach directions using Training (naive, test) $\times$ Phase (early, middle, late) $\times$ Cycles (cycle $1 \ldots 20)$ ANCOVAs, with prerotation percent adaptation entered as covariates. Greenhouse-Geisser corrections were applied where appropriate. For Experiment 1, test block adaptation was compared with naive block adaptation within the same group of participants. For Experiment 2, test block adaptation was compared with the training block of Experiment 2 with equivalent task error manipulations. Specifically, the StdTE ${ }^{\text {Train }}$ NoTE $^{\text {TEST }}$ group and the RandomTE ${ }^{\text {Train }}$ NoTE ${ }^{\text {TEST }}$ group experienced no task errors at test, and thus were compared with the Training block of the NoTE ${ }^{\text {Train }}$ StdTE $^{\text {TEST }}$ group who were first experiencing the rotation under no-task-error conditions. The NoTE ${ }^{\text {Train }}$ StdTE ${ }^{\text {TEST }}$ group experienced standard task errors at test and thus were compared with the Training block of the StandardTE Train NoTE ${ }^{\text {TEST }}$ group who were first experiencing the rotation under standard-task-error conditions. For Experiment 3, test block adaptation with standard task errors was compared with a different naive standard task error group (NaiveStandardTE ${ }^{\exp 3}, n=12: 6 \mathrm{CW}, 6 \mathrm{CCW}$ ). For Experiment 4, test block adaptation with short preparation time and standard task errors were compared with a naive control group (ShortNaive) who experienced the same short preparation time and standard task error conditions, but were naive to any training to reduce task errors or SPEs.

A common alternative measure of savings is to assess rate constants obtained by fitting the data to exponential functions. Rate constant analyses were not used here for the following reasons. For datasets where savings is evident as immediate adaptation in the first cycle upon perturbation exposure (Landi et al., 2011; Huberdeau et al., 2015), rate constants are typically small because the rapid initial adaptation is not captured by the fit. This can give the erroneous impression that savings is 
absent, and this situation was apparent in some of our data. When we tried to avoid this problem by fixing the fit parameter that reflects the $y$ value when $x=0$ as the mean reach direction in the immediately previous no-rotation cycle, the fits poorly characterized the data. These results agree with previous work demonstrating that exponential functions poorly represent individual learning curves, which often show abrupt step-like increases in performance (Gallistel et al., 2004).

Statistical analyses were performed with SPSS (IBM) and JASP. Graphs were plotted with Prism version 7.00 for Windows (GraphPad Software).

\section{Results}

\section{Experiment 1: task errors are important for savings}

In Experiment 1, we asked whether savings would be present if adaptation was learnt in the absence of task errors, or with task errors that were enforced regardless of the participants' actions. During training, all participants were exposed to a $30^{\circ}$ rotation of cursor feedback. In the StandardTaskError condition (StandardTE ${ }^{\text {Train\&Test }}$ ), since the target was not moved within a trial, task errors were allowed to vary contingent upon participants' responses to the cursor perturbation (Fig. 1B). Task errors were enforced in the random task error group (RandomTE $^{\text {Train\&Test }}$ ) condition by moving the target midmovement so that the cursor always missed the target by $20^{\circ}-30^{\circ}$ (these jumps were half CW, half CCW, in random order) (Fig. $1 E)$. Task errors were removed in the NoTaskError condition (NoTE ${ }^{\text {Train\&Test }}$ ) by moving the target to align with the cursor trajectory mid-movement (Fig. $1 H$ ). After initial exposure to the visuomotor rotation, behavior was returned to the unadapted state by removing the cursor rotation in a washout phase. During this washout, we also used the NoTaskError manipulation across all groups to prevent experience of task errors upon abrupt removal of the cursor rotation. At the test block (i.e., the second exposure to the visuomotor rotation), we applied the same task error manipulations that each group experienced when they were initially exposed to the perturbation.

Figure $1 C, F, I$ shows percent adaptation in the second exposure to visuomotor rotation compared with naive. With standard task errors, mean percent adaptation at test improved compared with naive, particularly in the first few cycles of the early adaptation phase (Fig. 1C), as Block $\times$ Phase $\times$ Cycle ANCOVA (preperturbation percent adaptation as covariate) showed a Block $X$ Phase $\times$ Cycle interaction $\left(F_{(38,342)}=3.02, p=4.5 \times 10^{(-8)}\right.$, partial $\left.\eta^{2}=0.25\right)$. As savings with standard task error conditions tends to be most prominent in the first few cycles, we followed this up with a Block $\times$ Cycle ANCOVA on the first 5 cycles (preperturbation percent adaptation as covariate), which showed a Block $\times$ Cycle interaction $\left(F_{(4,36)}=2.9, p=0.035\right.$, partial $\eta^{2}=$ $0.03)$, as mean adaptation in the first 5 cycles $(54.2,95 \%$ CI [36.9, $71.5])$ was better than naive $(34.3 \%, 95 \%$ CI $[17,51.6])$.

With random task errors (Fig. 1C), percent adaptation did not differ reliably between blocks across all phases and cycles, as Block $\times$ Phase $\times$ Cycle ANCOVA (preperturbation percent adaptation as covariate) showed no reliable main effect of block $(p=0.225)$ and no reliable interactions with block (all $p>0.3)$.

With no task errors (Fig. $1 I$ ), percent adaptation at test tended to be worse-than-naive, as Block $\times$ Phase $\times$ Cycle ANCOVA (preperturbation percent adaptation as covariate) showed a significant main effect of block $\left(F_{(1,9)}=6.552, p=0.031\right.$, partial $\eta^{2}$ $=0.42)$ and a significant Block $\times$ Phase interaction $\left(F_{(2,18)}=\right.$ $4.815, p=0.021$, partial $\left.\eta^{2}=0.34\right)$. Worse-than-naive adaptation tended to occur at the middle phase (training $=66.3 \%, 95 \%$ CI $[55.2,77.3]$, test $=59.4 \%, 95 \%$ CI $[48.3,70.4])$ as well as the late phase $($ training $=70.8 \%, 95 \%$ CI $[59.8,81.9]$, test $=62.7 \%$, 95\% CI [51.6, 73.7]).

Thus, despite exposure to the same cursor perturbation, and therefore previous experience of similar SPEs, savings was not evident in the groups that did not experience correctable task errors as a result of the cursor perturbation.

\section{Experiment 2: task errors are required at encoding but not at retrieval}

The absence of savings when perturbation-induced task errors were removed suggests some role of perturbation-induced task errors in savings. A few interpretations are possible. First, task errors might act as a retrieval cue to trigger the memory that is responsible for savings (Huberdeau et al., 2015). Second, task errors might be necessary to encode a memory that is responsible for savings. Third, task errors might be necessary both at encoding and at retrieval for savings: savings can only occur when previously experienced task errors are revisited. We dissociated these possibilities in Experiment 2. Task errors were manipulated either at training or at test to identify whether savings requires prior experience of task errors during first exposure to a perturbation (when a memory is first "encoded") or when the perturbation is reencountered (when a memory is "retrieved") (Fig. 2).

Specifically, a StandardTE ${ }^{\text {Train }}$ NoTE $^{\text {TEST }}$ group $(n=12: 6$ CW, 6 CCW) was deprived of task errors at test (target was shifted mid-movement so that the cursor always hit the target) but was provided standard task errors at training (i.e., no target shifts) (Fig. 2B): absence of improved adaptation here would suggest that task errors are necessary as a retrieval cue for savings. A NoTE $^{\text {Train }} \operatorname{StdTE}^{\text {TEST }}$ group $(n=12: 6 \mathrm{CW}, 6 \mathrm{CCW})$ was deprived of task errors at training, but experienced standard task errors at test (target did not move mid-movement) (Fig. 2H): absence of improved adaptation here would suggest that the task errors are not required as a retrieval cue but are a necessary component to encoding a memory that results in savings. Does improved adaptation result from the experience of task errors alone, or does it require learning to correct for task errors? To test this, a RandomTE ${ }^{\text {Train }}$ NoTE $^{\text {TEST }}$ group $(n=12: 6 \mathrm{CW}, 6 \mathrm{CCW})$ were provided with enforced but random task errors at training (target always moves away from the cursor mid-trial by 20-30 degrees, half in the CW direction, half in the CCW direction, such that they could never succeed in reducing task errors), and were tested for improved adaptation in the absence of task errors (Fig. 2E). After training, all groups encountered 6 no-feedback cycles and 60 no-rotation washout cycles with no task error, and then reencountered the same cursor rotation as they experienced at training.

To evaluate how adaptation with correctable and uncorrectable task errors at training affected subsequent adaptation under no-task-error conditions, we compared no-task-error test phase data of the StandardTE ${ }^{\text {Train }}$ NoTE $^{\text {TEST }}$ and the RandomTE ${ }^{\text {Train }}$ NoTE ${ }^{\text {TEST }}$ groups with the no-task error naive training block from the NoTE ${ }^{\text {Train }}$ StdTE ${ }^{\text {TEST }}$ group. This was done by running a Condition (naive training block of the NoTE ${ }^{\text {Train }}{ }^{2} \operatorname{StTE}^{\mathrm{TEST}}$ group, test block of the StandardTE ${ }^{\text {Train }}$ NoTE $^{\text {TEST }}$ group, test block of the RandomTE ${ }^{\text {Train }}$ NoTE $^{\text {TEST }}$ ) $\times$ Phase (early, middle, late) $\times$ Cycle (1. . 20) ANCOVA (prerotation percent adaptation as covariate). There was a significant main effect of Condition $\left(F_{(2,32)}=11.6, p=1.6 \mathrm{e}-4\right.$, partial $\left.\eta^{2}=0.4\right)$ as mean percent adaptation averaged across entire adaptation block for the StandardTE ${ }^{\text {Train }}$ NoTE $^{\text {TEST }}$ group $(75.1 \%$, 95\% CI [65.3, 85.0]) was better than naive (naive training block from NoTE $^{\text {Train }}{ }^{2} \operatorname{stdE}^{\mathrm{TEST}}, 40.6 \%$, 95\% CI $[29.0,52.2], t=4.7$, 


\section{A}

\section{Experiment 1}

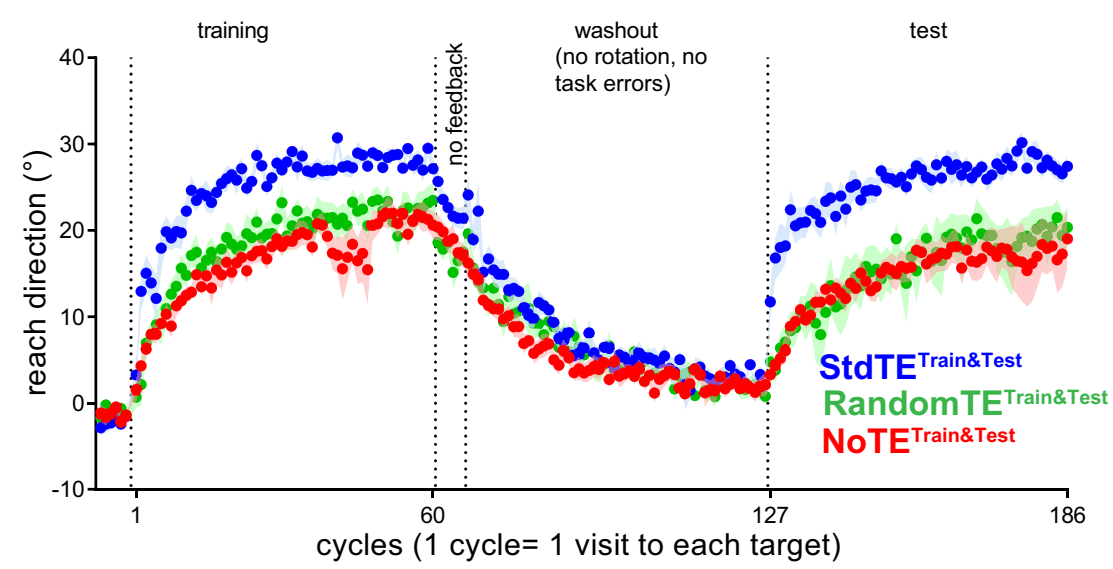

Training and test with standard task errors (StdTE Train\&Test)

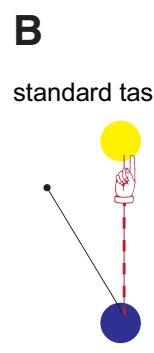

Training
C

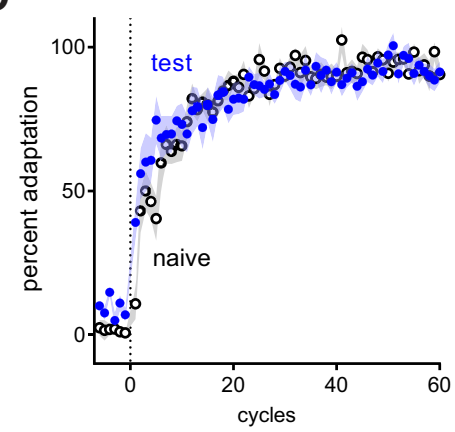

Training and test with random task errors (RandomTE Train\&Test)

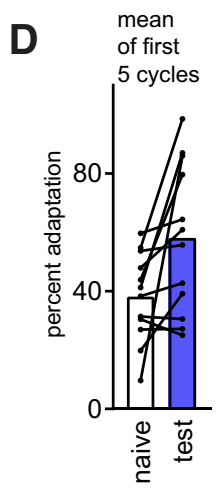

E

random task errors random task errors 20-30 (half CW/ half $20-30^{\circ}$ (half CW/ half CCW, randomly)

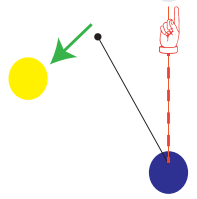

Training $\mathrm{CCW}$, randomly)

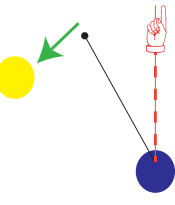

Test
$\mathbf{F}$

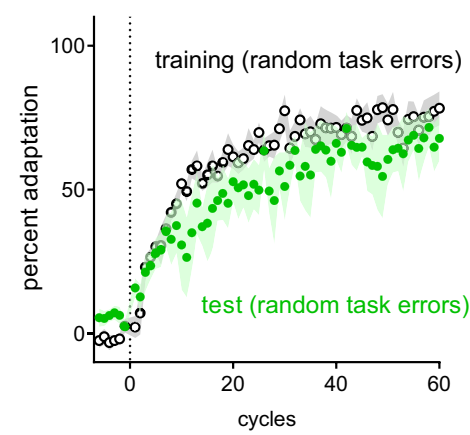

G $\quad \begin{gathered}\text { mean } \\ \text { of first }\end{gathered}$

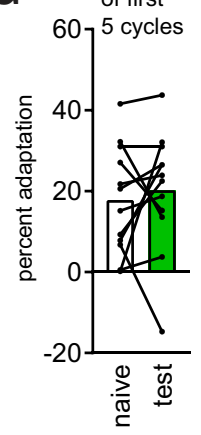

Training and test with no task errors (NoTE ${ }^{\text {Train\&Test) }}$

H

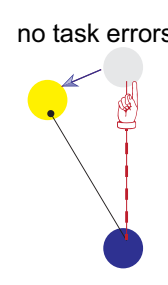

Training
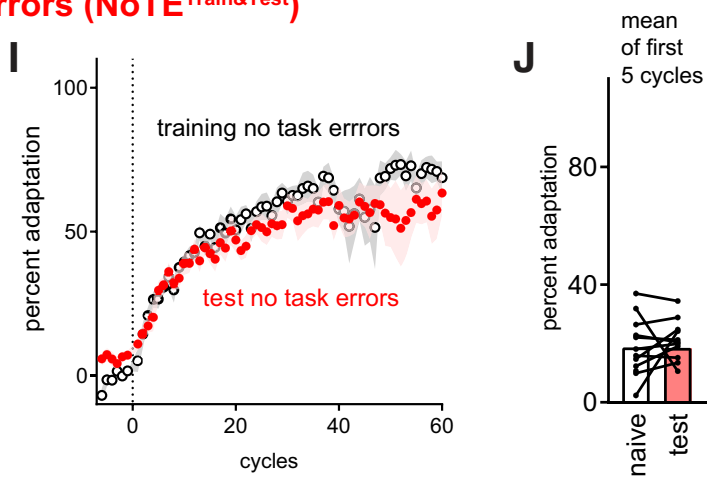

Figure 1. Experiment 1: Despite reexposure to the same rotation, savings was absent with no task errors or random task errors. $A$, Mean reach directions \pm SEM for all cycles for all groups (groups experiencing (W rotations sign-flipped to collapse across (W and CCW groups). One participant from the no task error group did not move toward the presented target in the cycles 41-47, which resulted in the variability that is apparent in the group average plot. Analysis outcomes were similar with and without this dataset. $\boldsymbol{B}, \boldsymbol{E}, \boldsymbol{H}$, Task error manipulations. $\boldsymbol{C}, \boldsymbol{F}, \boldsymbol{I}$, Estimated marginal mean \pm SEM of percent adaptation (covariate adjusted for prerotation percent adaptation). $\boldsymbol{D}, \boldsymbol{G}, \boldsymbol{J}$, Mean percent adaptation averaged from the first 5 cycles. 


\section{Experiment 2}

A

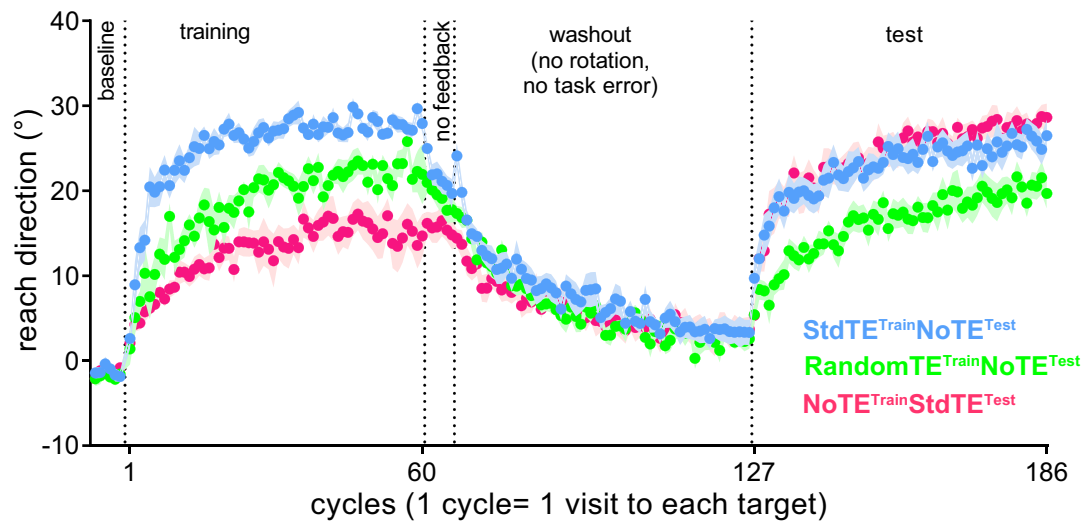

TrainStandardTaskErrors, TestNoTaskErrors (StdTE ${ }^{\text {Train }}$ NoTE $^{\text {Test}}$ )

B

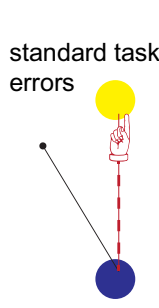

Training
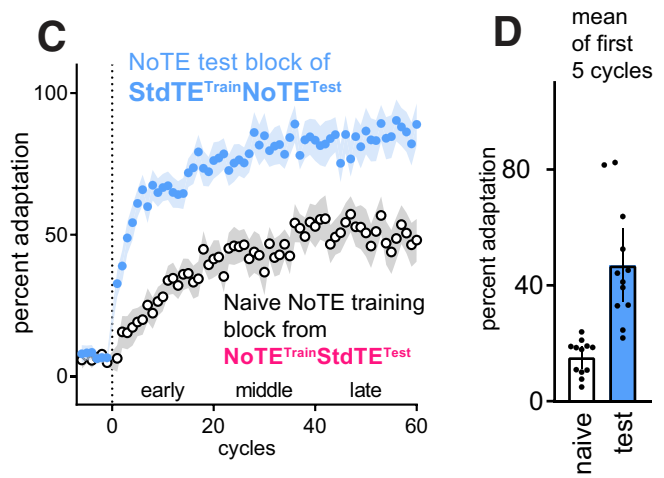

TrainRandomTaskErrors, TestNoTaskErrors (RandomTETrain NoTE ${ }^{\text {Test}}$ )

E
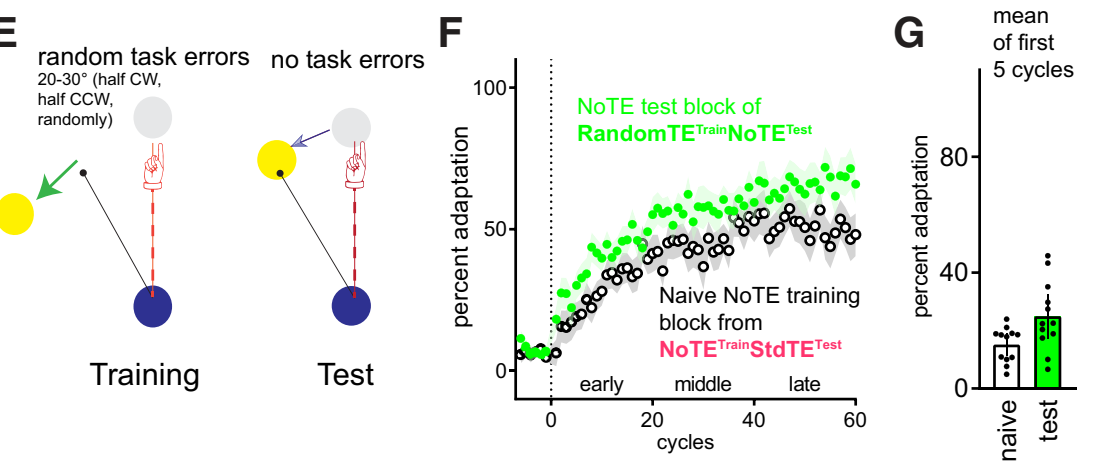

TrainNoTaskErrors, TestStandardTaskErrors (NoTE ${ }^{\text {Train }}$ StdTE $^{\text {Test }}$ )
H

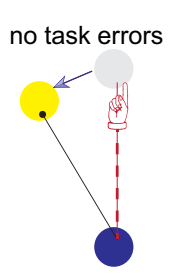

Training

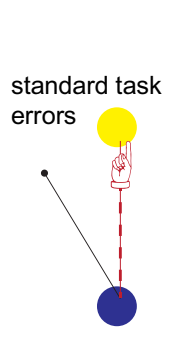

Test
I

Naive StdTE training block from StdTETrain NoTE Test

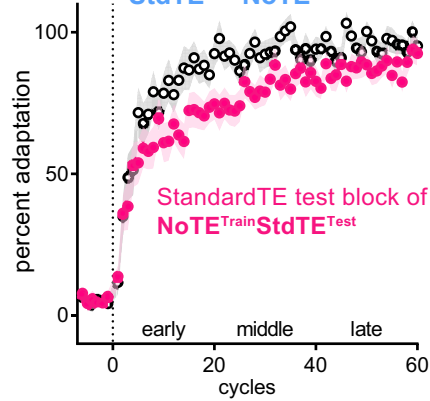

J mean 5 cycles

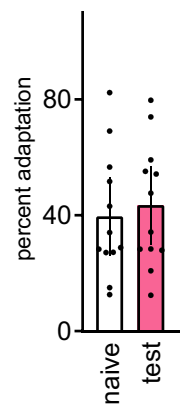

Figure 2. Experiment 2 showed that improved adaptation requires a history of adaptation to task errors. $\boldsymbol{A}$, Mean reach directions for all cycles for all groups (groups experiencing (W rotations sign-flipped to collapse across (W and CCW groups). $\boldsymbol{B}$, The StandardTE ${ }^{\text {Train }}$ NoTE ${ }^{\text {TEST }}$ group experienced standard task errors at training, but no task errors at test. $\boldsymbol{E}$, The RandomTE Train
Cohen's $\left.d=0.8, \mathrm{p}_{\text {bonf }}=1.3 \mathrm{e}-4\right)$. Previous adaptation with random task errors did not significantly improve adaptation at test (RandomTE ${ }^{\text {Train }}$ NoTE $^{\text {TEST }} 53.3 \%$, 95\% CI $[43.2,63.4])$ compared with the naive training block of $\mathrm{NoTE}^{\text {Train }} \mathrm{Std}$ $\operatorname{TE}^{\mathrm{TEST}}(40.6 \%, 95 \% \mathrm{CI}[29.0,52.2], t=$ 1.8 , Cohen's $\left.d=0.3, \mathrm{p}_{\text {bonf }}=0.3\right)$. The StandardTE ${ }^{\text {Train }}$ NoTE $^{\text {TEST }}$ group showed better adaptation than the RandomTE ${ }^{\text {Train }}$ NoTE ${ }^{\text {TEST }}$ group $(t=3.2$, Cohen's $d=$ $\left.0.5, \mathrm{p}_{\text {bonf }}=9.5 \mathrm{e}-3\right)$. Thus, a history of adapting to task errors resulted in betterthan-naive test block adaptation to a cursor rotation, even when the perturbation did not cause any task errors upon second exposure. In the random task error condition, merely experiencing task errors, without learning to correct for those task errors, was not sufficient to improve adaptation.

To evaluate whether the presence of task errors at test is necessary to retrieve previous adaptation to SPEs to subsequently improve adaptation compared with naive, we compared the test block of the NoTE ${ }^{\text {Train }}$ StdTE ${ }^{\text {TEST }}$, to the training block from the StandardTE ${ }^{\text {Train }}$ NoTE ${ }^{\text {TEST }}$ group where participants first encountered the rotation under standard task error conditions. Fig. $2 D$ suggests that adaptation did not improve compared with naive. Condition (naive training block of the StandardTE ${ }^{\text {Train }}$ NoTE $^{\text {TEST }}$ test block of the NoTE ${ }^{\text {Train }}$ StdTE $^{\text {TEST }}$ group) $\times$ Phase (early, middle, late $) \times$ Cycle ANCOVA with prerotation baseline as covariate showed a significant Phase $X$ Cycle $\times$ Condition interaction $\left(F_{(38,798)}\right.$ $=1.921, p=8.329 \mathrm{e}-4$, partial $\eta^{2}=$ $0.084)$. Mean percent adaptation averaged across all cycles tended to be less in the NoTETrainStdTETEST group (78.9\%, $95 \%$ CI $[70.8,86.9])$ than naive $(89.6 \%$, $95 \%$ CI $[80.1,99.2])$. Thus, depriving participants of task errors when they were first exposed to the cursor rotation at training did not improve subsequent ad-

\footnotetext{
NoTE ${ }^{\text {TEST }}$ group experienced random task errors at training but no task errors at test. $\boldsymbol{H}$, The NoTE Train StdTE TEST group experienced no task errors at training, and standard task errors at test. All participants were deprived of task errors (i.e., had the no-task error manipulation) at washout. $\boldsymbol{C}, \boldsymbol{F}, \boldsymbol{I}$, Estimated marginal mean \pm SEM adjusted for prerotation percent adaptation, where test phase adaptation is compared with naive controls (open symbols) experiencing the same task error manipulation. A history of adapting to standard task errors, but not adapting to random task errors, improved subsequent adaptation $(\boldsymbol{C}, \boldsymbol{F})$. In contrast, adaptation was unimproved without a history of task errors (I). D, G,J, Mean $\pm 95 \%$ Cl of mean percent adaptation averaged from the first 5 cycles.
} 
aptation, despite the presence of standard task errors.

Thus, a history of adapting movements to correct task errors appears necessary to encode learning that improves adaptation to a previously experienced visuomotor rotation. The presence of task errors appears unnecessary to retrieve this learning. All groups with ample prior exposure to SPEs but who had no prior experience of correcting task errors did not show improved subsequent adaptation (Experiment 1: RandomTE ${ }^{\text {Train\&Test }}$, NoTE ${ }^{\text {Train\&Test }}$; Experiment 2: RandomTE ${ }^{\text {Train }}{ }^{\text {NoTE }}{ }^{\text {TEST }}$ and NoTE $^{\text {Train }}$ StdTE $^{\text {TEST }}$ ). Thus, a history of SPEs appears to be insufficient to improve subsequent adaptation.

\section{Experiment 3: learning to correct for task errors is sufficient to evoke savings}

Recent work suggests that savings in visuomotor rotation primarily reflects the deliberate application of a strategy, where participants explicitly re-aim to the one side of a target to counteract the rotation of cursor feedback (Haith et al., 2015; Morehead et al., 2015). This view considers the role of implicit adaptation to SPEs as secondary to the role of strategy in savings, and would interpret the presence/absence of savings with task errors in Experiments 1 and 2 to be because task errors provoke the formation of explicit strategies. An alternative view is that task errors alter the sensitivity to SPEs (Leow et al., 2018; Kim et al., 2019), and increased sensitivity to these errors produces savings (Herzfeld et al., 2014). We cannot dissociate between the two alternative explanations based on data from Experiments 1 and 2 alone because task errors here were not wholly independent of SPEs; the task error manipulations were always made in the presence of a perturbation of the hand cursor relationship (which therefore induced SPEs). Thus, we next examined how learning to correct for task errors alone, in the absence of SPEs (i.e., in the absence of any perturbation of the hand cursor relationship), affected subsequent adaptation to a visuomotor rotation.

In Experiment 3, we did not perturb the cursor at training but enforced systematic task errors that could be counteracted by a re-aiming strategy: the target always moved away by $30^{\circ}$ from the original target location mid-movement: participants could correct these task errors by re-aiming $30^{\circ}$ away from the original target (Fig. 3A). For one group of participants, the reach solution needed to hit a given target after it jumped mid-movement was the same reach solution needed to counteract the cursor rotation in the test block (Same, $n=12: 6 \mathrm{CW}, 6 \mathrm{CCW}$; Fig. $3 B$ ). For example, if the target jump at training was $30^{\circ} \mathrm{CCW}$, then the cursor rotation at test was $30^{\circ} \mathrm{CW}$ (thus requiring a CCW compensatory hand movement). To test whether this learning is flexible enough to accommodate a different reach solution, we had another group of naive participants (Different, $n=12: 6 \mathrm{CCW}, 6$ $\mathrm{CW})$, where the reach direction required to hit targets at training was opposite to that at test (Fig. 3E). Pilot testing revealed substantial individual differences in how quickly participants developed a strategy to re-aim at training. Thus, if the experimenter observed that participants had yet to show successful re-aiming by trial 180 of the 480-trial training block, the experimenter explicitly instructed participants that a strategy may be needed to hit the target. This explicit instruction was required in 2 of the 12 participants in the Same group and 6 of the 12 participants in the Different group. Here, the instruction was given without exposure to the cursor perturbation but after exposure to the task error. At the test block, no instructions about re-aiming were provided. To quantify improved adaptation, we compared percent adaptation at test with a different naive control group who experienced similar task error manipulations $(\mathrm{Na}-$ iveStandardTE ${ }^{\exp 3}, n=12: 6 \mathrm{CW}, 6 \mathrm{CCW}$ ).

Figure $3 A$ shows reach directions in all cycles. After instructing participants that the task error manipulation had been removed, reach directions reverted rapidly back to baseline in the no-feedback block. This illustrates that the re-aiming response can be switched off immediately upon instruction, similar to previous work (e.g., Mazzoni and Krakauer, 2006; Benson et al., 2011; Taylor and Ivry, 2011; Schween et al., 2014; Savoie et al., 2018).

Despite being naive to the cursor rotation, improved adaptation was evident when the reach solution at test was the same as training, and when the reach solution at test was different from training (Fig. 3C,F). A three-way ANCOVA [Condition (Same, Different, Naive StdTEexp3 $) \times$ Phase (early, middle, late $) \times$ Cycle $1 \ldots 20$, with prerotation percent adaptation as covariate] showed a significant Phase $\times$ Condition interaction $\left(F_{(4,19)}=\right.$ 4.541, $p=0.003$, partial $\left.\eta^{2}=0.22\right)$ and a significant Condition $\times$ Phase $\times$ Cycles interaction $\left(F_{(24.9,399.5)}=2.63, p=7.101 \mathrm{e}-12\right.$, partial $\left.\eta^{2}=0.14\right)$. As savings tested in standard task error conditions tends to be most prominent in the first few cycles, we followed this up with a Condition (Same, Different, Naive StdTEexp3 $\left._{\text {S }}\right) \times$ Cycles ANCOVA on the first 5 cycles, which showed a significant main effect of Condition $\left(F_{(4,128)}=9.629, p=5.323 \mathrm{e}-4\right.$, partial $\eta^{2}=$ $0.376)$ and a Cycles $\times$ Condition interaction $\left(F_{(4,128)}=2.759\right.$, $p=0.008$, partial $\left.\eta^{2}=0.147\right)$. Both the Same and the Different group showed better-than-naive percent adaptation averaged across the first 5 cycles: NaiveStdTE $\exp _{3}=32.2 \%,[18.2 \%, 46.3 \%]$ versus Same $(69.8 \%,[55.7 \%, 83.8 \%], t=3.59$, Cohen's $d=$

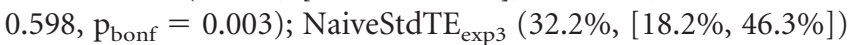
versus Different (72.4\%, [58.4\%, 86.4\%], $t=3.996$, Cohen's $d=$ 0.666 , pbonf $=0.001)$. We note that the result of the Different group showing improved adaptation at test stands in contrast to a previous finding of interference from previously learning an incongruent reach solution (Zach et al., 2005), but in that study, neither washout, nor task instruction, nor change in task context was given to participants to disengage the previous reach solution: interference there might be because participants unwittingly continued behaving as before.

Hence, previous learning to counteract task errors was sufficient to improve subsequent adaptation to a visuomotor rotation, even when participants were naive to perturbation-induced SPEs. This learning was flexible: it elicited improved adaptation with respect to naive performance, even when the reach solution required to hit a given target at training was different from the reach solution required to hit that target at test.

\section{Experiment 4: suppressing goal-directed behavior by reducing movement preparation time}

We next explored the mechanisms by which learning to compensate for task errors can improve subsequent visuomotor rotation adaptation. Task error correction likely relies on strategic, goaldirected processes that demand cognitive resources because error compensation is reduced by manipulations that limit cognitive resources, such as time constraints or dual-tasking (Taylor and Thoroughman, 2007, 2008; Galea et al., 2010; Malone and Bastian, 2010; Fernandez-Ruiz et al., 2011; Anguera et al., 2012; Haith et al., 2015; Leow et al., 2017). In visuomotor rotation paradigms, these observations are consistent with the notion that mental rotation of a movement plan at a specified angle away from a target is computationally expensive and requires time (Georgopoulos and Massey, 1987; Pellizzer and Georgopoulos, 1993; Bhat and Sanes, 1998). Does savings solely result from goal- 
A Experiment 3

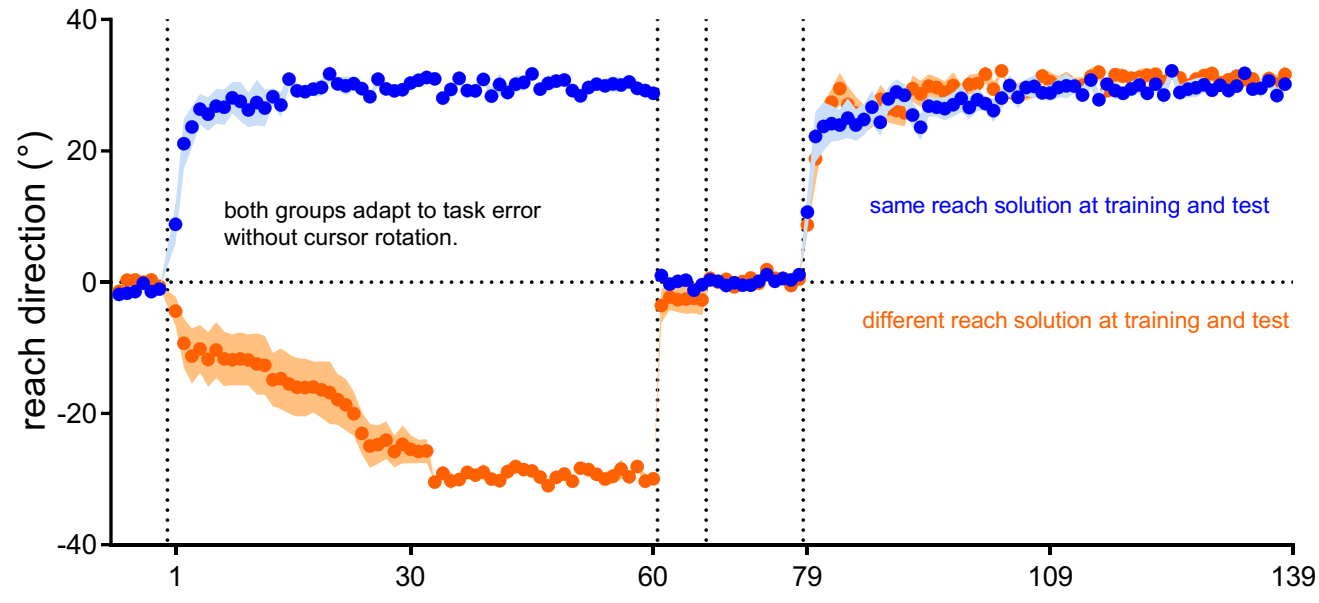

Train with same reach solution but no cursor rotation, test with standard task errors

B task error, no cursor rotation

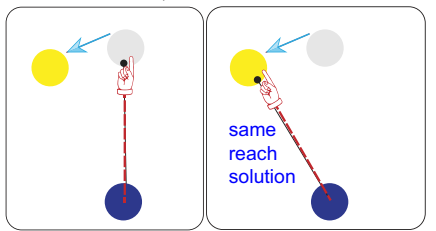

Test: cursor rotation requires same reach solution as training

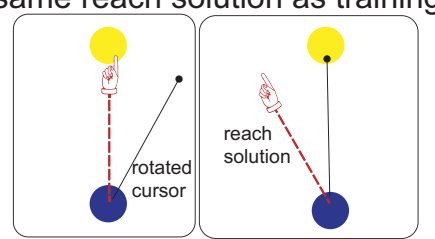

C

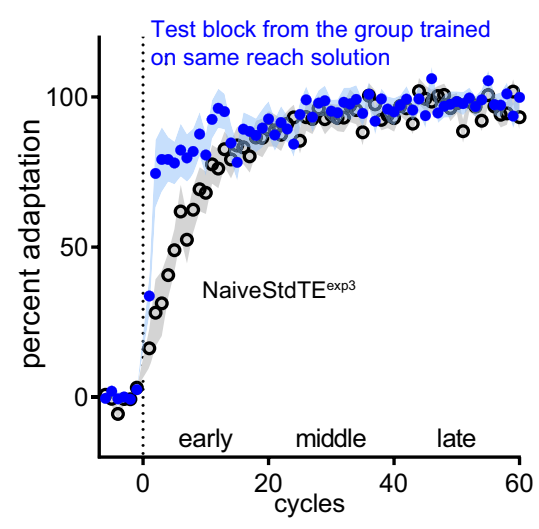

D mean

of first 5 cycles

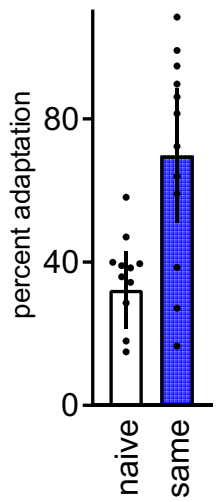

Train with different reach solution but no cursor rotation, test with standard task errors

E Training: systematic task error, no cursor rotation

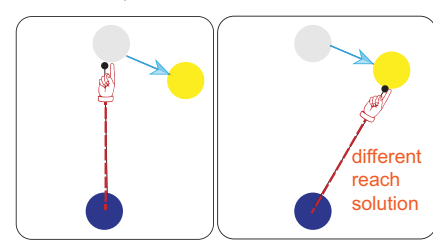

Test: cursor rotation requires different reach solution as training

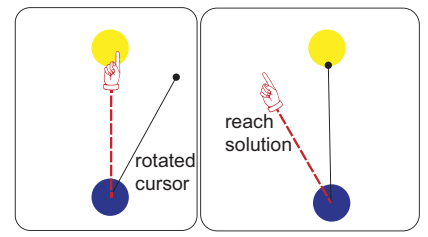

$\mathbf{F}$

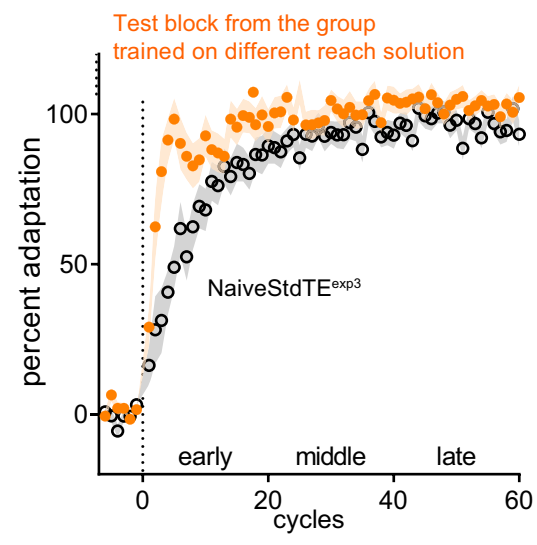

G

$$
\begin{aligned}
& \text { mean } \\
& \text { of first }
\end{aligned}
$$

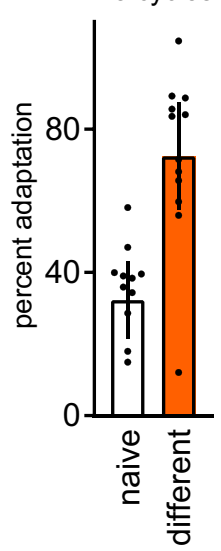

Figure 3. Experiment 3 tests how experience correcting task errors without cursor rotation-induced sensory prediction errors affects subsequent adaptation to cursor rotation. At training, the target moved from its original position by $30^{\circ}$ mid-movement (cursor was not rotated): participants learnt to counteract task errors by moving the cursor $30^{\circ}$ away from the presented target. Participants promptly disengaged this behavour upon instructions of disturbance removal $(\boldsymbol{A})$. At test, participants encountered a $30^{\circ}$ cursor rotation for the first time. The Same group had the same reach solution at test as at training (see $\boldsymbol{B}$ ). The Different group had a directionally opposite reach solution at training and at test (see $\boldsymbol{E}$ ). (\&F: Estimated marginal means \pm SEM adjusted for pre-rotation percent adaptation in both groups showed better-than-naïve adaptation. D\&G: mean $\pm 95 \% \mathrm{Cl}$ of mean percent adaptation averaged from the first 5 cycles. 
directed cognitive strategies (Morehead et al., 2015)? To test this, we examine whether improved adaptation occurred when these time-consuming cognitive processes were suppressed by limiting preparation time. We replicated the design of Experiment 3, except that participants were required to move within a short preparation time of $250 \mathrm{~ms}$ after the time of target presentation throughout all trials. We used the timed-response paradigm described in Materials and Methods for all the previous experiments, but instead of presenting the target at $1000 \mathrm{~ms}$ before the imperative to move, in Experiment 4 we presented the target 250 ms before the imperative to move (Leow et al., 2017). There were two conditions: ShortSame $(n=12: 6 \mathrm{CW}, 6 \mathrm{CCW})$, where the reach solution is the same at training and at test, and ShortDifferent, $(n=12: 6 \mathrm{CW}, 6 \mathrm{CCW})$, where the reach solution is opposite at training and at test. Explicit instructions to re-aim were necessary in 4 of the 12 participants in the ShortSame group, and 8 of the 12 participants in the Different group. Test phase adaptation was compared with a naive control group ShortNaive $(n=12: 6 \mathrm{CW}, 6 \mathrm{CCW})$.

At training, despite the preparation time constraints, participants did learn to compensate for task errors (Fig. 4A), as percent adaptation was larger at the late phase of the training block than the early phase. However, the extent of compensation at training (estimated as the last 10 cycles) was less complete with short preparation time in Experiment 4 (ShortDifferent $=54.8 \%, 95 \%$ CI $[47.9,61.6]$, ShortSame $=50.5 \%, 95 \%$ CI $[43.6,57.5])$ than with long preparation time in Experiment 3 (Different $=97.4 \%$, 95\% CI [90.6, 104.2]; Same $=98.2 \%, 95 \%$ CI $[91.4,105.1])$, as Preparation Time (Short, Long) $\times$ Training Direction $\times$ Cycles (Adapt51 . . 60) ANCOVA (with prerotation percent adaptation entered as covariate) showed a significant main effect of preparation time $\left(F_{(1,43)}=176.17, p=0\right.$, partial $\left.\eta^{2}=0.8\right)$.

To evaluate how test phase adaptation was affected by prior training with a reach solution that was the same or different from that needed to counteract the test phase cursor rotation, we ran a Condition (ShortSame, ShortNaive, ShortDifferent) $\times$ Phase $\times$ Cycles ANCOVA (with prerotation percent adaptation as covariate) on all adaptation cycles. There was a significant Phase $\times$ Condition interaction $\left(F_{(3,48.8)}=9.77, p=0\right.$, partial $\left.\eta^{2}=0.37\right)$ and a significant Condition $\times$ Phase $\times$ Cycles interaction $\left(F_{(27.0,433.3)}=1.73, p=0.013\right.$, partial $\left.\eta^{2}=0.09\right)$. Follow-up Condition $\times$ Cycle ANCOVAs run on the first 5 cycles showed a significant main effect of Condition $\left(F_{(2,32)}=21.15, p=\right.$ 0.000001 , partial $\left.\eta^{2}=0.56\right)$. In these first 5 cycles, the ShortSame group showed better-than-naive adaptation (ShortNaive = $26.2 \%, 95 \%$ CI $[18.8,33.5]$; ShortSame $=43.9 \%, 95 \%$ CI $[36.5$, $51.2], t=3.474$, Cohen's $d=0.579$, p bonf $=0.004)$ whereas the ShortDifferent group showed worse-than-naive adaptation (ShortNaive $=26.2 \%$, ShortDiff $=10.7 \%, 95 \%$ CI $[3.4,18.1], t=$ 3.027, Cohen's $d=0.504$, $\mathrm{p}_{\text {bonf }}=0.015$ ).

\section{Does adaptation extent mediate the effect of task error conditions during training on improved test phase adaptation performance?}

We interpreted results of Experiments 1 and 2 to suggest that adaptation to task errors at training improved adaptation performance at test. An alternative possibility is that improved adaptation at test is determined by extent of adaptation at training, rather than by task error conditions at training. If the effect of task error condition on test phase adaptation results solely from its effects on adaptation extent, a mediation analysis would show that the effect of training phase task error conditions is fully mediated by the extent of training phase adaptation (i.e., the effect of task error condition at training would become nonsignificant when accounting for extent of extent of adaptation at training). Mediation analyses are conservative with respect to our hypothesis that task error conditions at training influence subsequent test phase adaptation. This is because we expect a strong relationship within individuals between training and test performance: thus, the mediator of adaptation extent at training is highly likely to account for some variance in test performance. Moreover, given the hypothesis that correction of task errors is necessary for savings, participants in the standard task error condition who adapted more upon first exposure to the perturbation might be expected to have better test performance than their peers because they made larger task-error driven corrections, rather than due to greater adaptation per se. Thus, partial mediation between initial adaptation magnitude and test performance is likely, even if the size of initial adaptation per se has no genuine effect.

Thus, we ran mediation analyses for Experiments 1 and 2 to examine whether the effect of training phase task error manipulation on test phase adaptation is fully mediated by the extent of adaptation in the training phase. Thus, we selected datasets for which the test phase task error conditions were the same from Experiments 1 and 2. As the task error manipulations at training were most sensitive in test conditions with no task errors, we ran our mediation analysis for all groups exposed to no-task errors at test: NoTE ${ }^{\text {Train\&Test }}$ (from Experiment 1), StdTE ${ }^{\text {Train }}{ }^{\text {NoTE }}{ }^{\text {Test }}$ (from Experiment 2), and Random ${ }^{\text {Train }}$ NoTE $^{\text {TEST }}$ (from Experiment 2). The mediation analyses requires that the dependent variable (test phase adaptation) is measured in the same way for all groups: in Experiment 1, the test phase is measured with different task error conditions for each group; in Experiment 2, the test phase was measured with the same task error condition for only the RandomTE ${ }^{\text {Train }}{ }^{\text {NoTE }}{ }^{\text {TEST }}$ and the Standard ${ }^{\text {Train }}$ NoTE ${ }^{\text {TEST }}$ groups. Thus, we only analyzed datasets for which the test phase task error conditions were the same. As the task error manipulations at training were most sensitive in test conditions with no task errors, we ran mediation analyses for all groups exposed to no-task errors at test: NoTE ${ }^{\text {Train\&Test }}$ (from Experiment 1), TrainStdTE ${ }^{\text {TEST }}$ NoTE $^{\text {Train }}$ (from Experiment 2), and Random $^{\text {Train }}$ NoTE $^{\text {TEST }}$ (from Experiment 2).

We used the nonparametric bootstrapping PROCESS macro version 3.4 in SPSS (IBM) for mediation analysis with 10,000 samples (Preacher and Hayes, 2004). This procedure has been recommended for samples between 20 and 80 (Shrout and Bolger, 2002). With samples $<80$, this procedure is biased toward the test hypothesis (Koopman et al., 2015). Our sample here was $n=36$ for each experiment: the analysis favors the test hypothesis that adaptation extent mediates the effect of task error conditions on improved test block performance (Koopman et al., 2015). Improvements compared with naive were estimated as the means of the first 5 cycles, but similar results were shown when the means of the first 20 cycles were used. The mediator variable of adaptation extent was quantified as the mean percent adaptation of the last 5 adaptation cycles. The covariate was mean percent adaptation in final 3 prerotation cycles (i.e., last 3 cycles of washout). Step 1 of the regression analysis confirmed that, before accounting for possible effects of the mediator, task error condition predicted test phase adaptation $\left(\mathrm{b}=10.583, t_{(33)}=4.416\right.$, $p=0.001)$. Step 2 showed that the task error condition predicted the mediator adaptation extent $\left(b=8.049, t_{(33)}=3.756, p=\right.$ $0.007)$. Step 3 showed that the mediator (adaptation extent) significantly predicted test phase adaptation, after controlling for task error condition $\left(\mathrm{b}=0.463, t_{(32)}=2.574, p=0.0149\right)$. Step 4 
A

\section{Experiment 4}

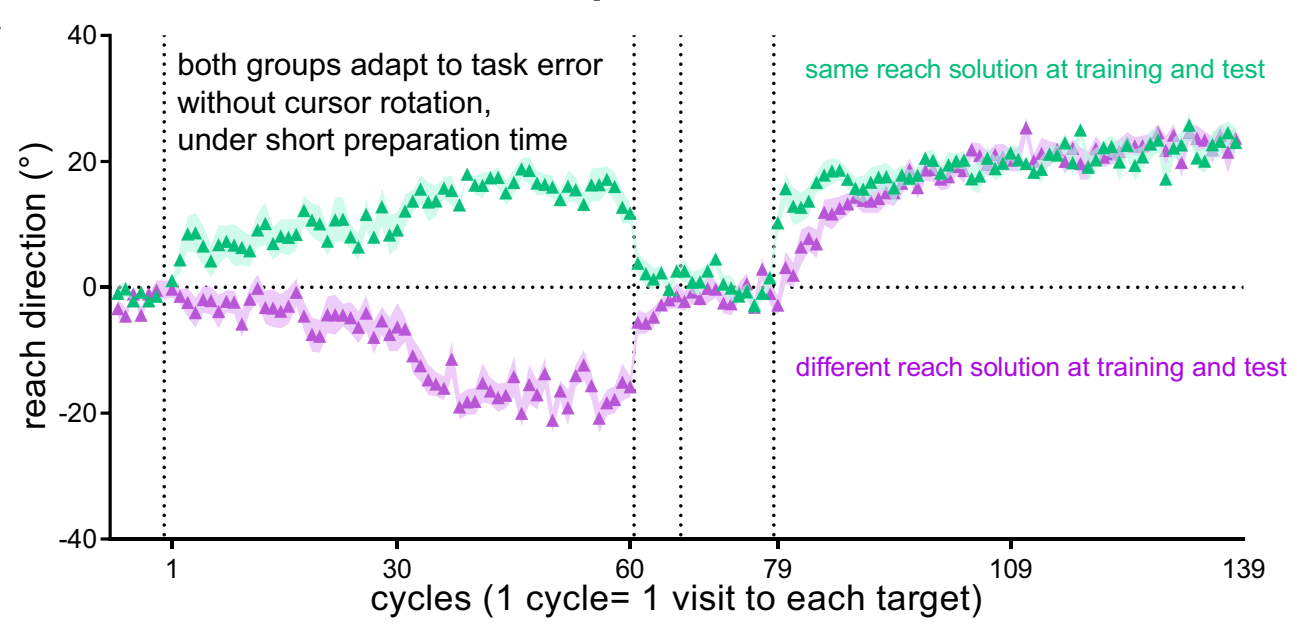

Train with same reach solution without cursor rotation, test with standard task error all trials under short preparation time conditions

B

Training: systematic task error, no cursor rotation

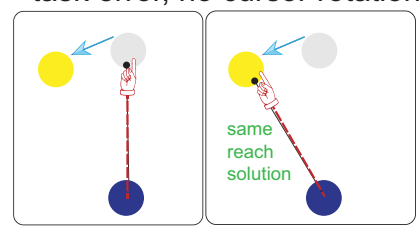

Test: cursor rotation requires same reach solution as training

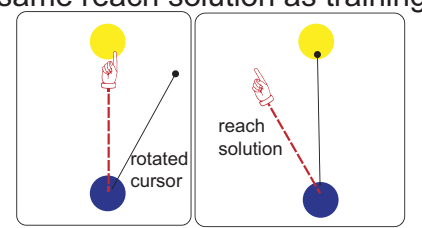

C

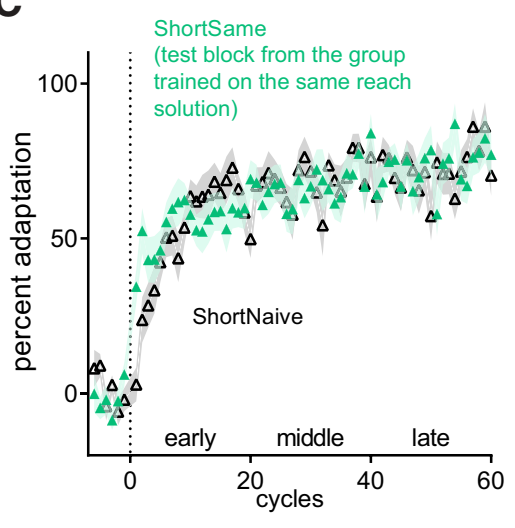

D mean 5 cycles

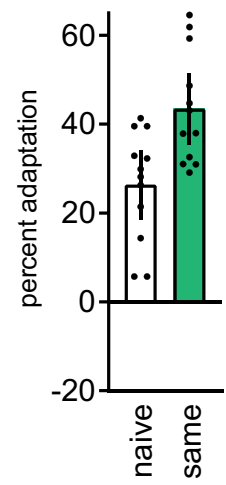

Train with same reach solution without cursor rotation, test with standard task error all trials under short preparation time conditions

E

Training: systematic task error, no cursor rotation

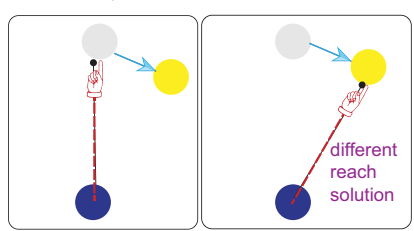

Test: cursor rotation requires different reach solution as training

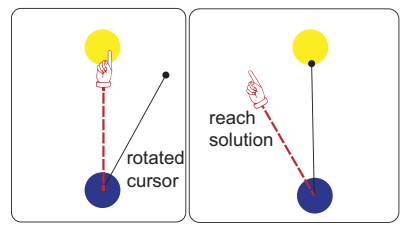

$\mathbf{F}$

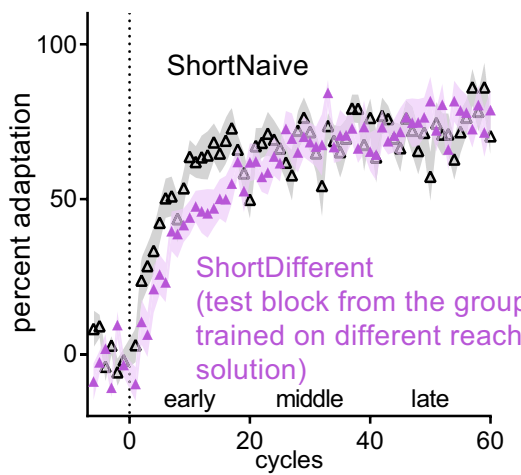

G mean 5 cycles

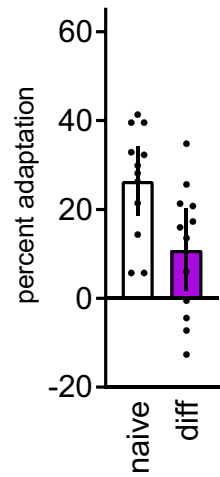

Figure 4. Experiment 4 design replicates Experiment 3 with either the same reach solution at test and training (i.e., ShortSame, $\boldsymbol{B}$ ), or a directionally opposite reach solution at training and at test (ShortDifferent, $\boldsymbol{E}$ ), but limiting amount of time available to prepare movement to $272 \mathrm{~ms}$ after target presentation. Mean \pm SEM for reach directions across all cycles $(\boldsymbol{A})$. Estimated marginal means \pm SEM of percent adaptation in the test phase (filled symbols), compared to naïve (clear symbols), for the ShortSame group $(\boldsymbol{C})$, or the ShortDifferent group $(\boldsymbol{F})$. Under short-preparation time, better-than-naive adaptation was evident in the ShortSame group (D) but not the ShortDifferent group (G). (D\&G = Means $\pm 95 \%$ Cl for first 5 cycles). 
showed that, after controlling for the mediator (adaptation extent), task error condition remained a significant predictor of test phase adaptation $\left(\mathrm{b}=6.855, t_{(32)}=2.590, p=0.0143\right)$. Thus, although the extent of adaptation at training partially mediated the effect of training block task error condition on early test block adaptation extent, the effect of training block task error condition remained significant after taking into account the mediating effect of adaptation extent. Hence, the extent of adaptation induced in the training phase cannot fully account for the relationship between task error conditions and test phase adaptation.

\section{Discussion}

Here, we demonstrate a fundamental role of adaptation to task errors in savings: a hallmark of latent sensorimotor memory. Savings was absent without prior correction of task errors (Experiment 1). Prior correction of task errors (Experiment 2), even when naive to perturbation-induced SPEs, was also sufficient to improve subsequent adaptation (Experiments 3 and 4). Thus, task errors that prompt adaptive responses can affect subsequent adaptation to never-before-encountered sensorimotor perturbations. Experiments 3 and 4 further demonstrate that these sensorimotor memories can rely on distinct processes: (1) a timeconsuming process that is flexible enough to facilitate corrective responses in the opposite direction; and (2) a rapidly accessible process that can be volitionally disengaged but is not sophisticated enough to produce a novel corrective response. Although better-than-naive adaptation shown in Experiments 2-4 does not strictly fulfill the criteria for "savings," as defined by improved readaptation to the same previously experienced perturbations, we believe that the forms of memory demonstrated by our experiments are likely to contribute to faster reacquisition of a previously extinguished sensorimotor memory when the tasks are the same (i.e., savings).

\section{Savings require prior adaptation to task errors}

Despite extensive study, exactly what mechanisms underpin savings remain unresolved. One influential theory suggests that a history of errors increases sensitivity to those errors, improving learning when reencountering familiar errors (Herzfeld et al., 2014). Sensorimotor perturbations typically evoke both SPEs and task errors, but it was previously unclear how these errors contribute to sensorimotor memories that improve learning (Orban de Xivry and Lefevre, 2015; Leow et al., 2016). Here we show that, at least in visuomotor rotation, a history of adaptation to task errors is crucial to encode memories that improve subsequent adaptation. Uncorrectable task errors (i.e., enforced, random task errors that occurred regardless of behavior) failed to evoke savings. Furthermore, task errors need not be present upon reexperiencing the perturbation, contradicting the idea that task errors act as a retrieval cue to trigger savings (Huberdeau et al., 2015). We note, however, that the absence of a measurable contribution of SPEs to savings in visuomotor rotation might not mean zero contribution of SPEs to all forms of persistent sensorimotor memories.

One alternative possibility to explain our finding that test phase performance was not enhanced when task errors at initial learning were either random or prevented, is learned helplessness. Learned helplessness occurs when there is noncontingency between response and reinforcement at training (e.g., when faced with a nonescapable electric shock, an unsolvable anagram, or when reward is yoked and unrelated to task performance), and the helpless behavior persists at test, even when the contingency between response and reinforcement is returned (e.g., failure to escape an easily escapable shock, solve an easily solvable problem, or obtain response-contingent reward) (Abramson et al., 1978). If our participants came to realize that there was nothing that they could do to influence task success or failure, they might have given up trying to hit the target, and this helplessness might have carried over to the test phase. This idea seems plausible in random task error conditions, when participants failed to hit targets regardless of what they did. In the no-task error conditions, participants succeeded in hitting targets regardless of where they reached. Some degree of learned helplessness can occur when reward is infrequent, randomly imposed, and uncorrelated with performance (Roth, 1980). Crucially however, learned helplessness does not occur in situations of $100 \%$ enforced success (i.e., reward in every trial) (Benson and Kennelly, 1976). Furthermore, noncontingency between response and reinforcement cannot explain the absence of savings under conditions with full contingency between response and reinforcement, but with gradually imposed perturbations which limited prior experience and correction of errors (Leow et al., 2016). It thus seems more parsimonious that deficient experience in correcting errors, rather than learned helplessness, was the culprit for the lack of improved adaptation in the groups who trained with random or no task errors.

\section{Improved adaptation does not require a history of SPEs}

When task errors were absent in Experiments 1 and 2, the lack of improved adaptation despite abundant prior exposure to SPEs suggests that the task errors, and not SPEs, are compulsory for savings. One possibility is that task errors drive deliberate corrective responses during adaptation, and that savings results from faster reselection of previously learnt actions (Haith et al., 2015; Huberdeau et al., 2015; Morehead et al., 2015). If true, then deliberate correction of task errors alone in the absence of SPEs should be sufficient for savings. We tested this in Experiment 3 by imposing a systematic task error at training (targets always jumped mid-movement by $30^{\circ}$ ) without perturbing hand position feedback. Learning to correct these task errors improved subsequent naive adaptation to the $30^{\circ}$ rotation of hand position feedback. Thus, learning to counteract task errors in the absence of a sensorimotor perturbation was enough to improve adaptation to a never-before encountered perturbation. However, improved adaptation was not merely due to faster reselection of previously learned actions, since we also observed adaptation benefits for different actions.

\section{How might correction of task errors lead to savings?}

What is encoded when correcting task errors? In Experiment 3, we found that adaptive responses to task errors can be volitionally disengaged, but this learning was retained in latent form to affect subsequent adaptation. Thus, this form of learning seems fundamentally distinct from adaptive responses to SPEs, which are expressed obligatorily after perturbation removal (Izawa and Shadmehr, 2011), indicating a remapping between motor intent and its predicted sensory outcomes. In contrast, the contextually flexible response to task errors might arise from an earlier component of the sensory-to-motor transformation: a mapping between the behavioral goal and the motor plan selected to achieve it (Crawford et al., 2011).

Given that we can instruct participants to deliberately use re-aiming strategies (e.g., Mazzoni and Krakauer, 2006), it is perhaps unsurprising that task errors led to savings by prompting acquisition of a re-aiming strategy. Indeed, one view is that savings results solely from deliberate strategy (Morehead et al., 
2015). This view is supported by the absence of savings (Haith et al., 2015) when time pressure is used to suppress time-consuming mental rotation processes required to re-aim accurately away from the presented target (e.g., Georgopoulos and Massey, 1987). Although recent findings show savings despite time pressure with repeated exposure to opposing visuomotor rotations (Huberdeau et al., 2019), savings in that study might have resulted partly from improvements in the residual capacity to re-aim away from a target at short latencies, when the range of required responses is small and predictable (Leow et al., 2017; McDougle and Taylor, 2019).

But is the capacity to use mental rotation the sole contributor to savings in visuomotor rotation? We explored this in Experiment 4 by replicating Experiment 3, but limiting preparation time. Targets were randomly presented in a wide spatial array, reducing predictability of the required response. With time pressure, reaches only partially compensated for the rotation at training (only $\sim 50 \%$ percent adaptation despite 480 training trials), consistent with previous work (Leow et al., 2017; McDougle and Taylor, 2019). At test, adaptation was better than naive when the reach solution at test matched that at training, and worse than naive when the reach solutions were opposite at training and test. Thus, although time pressure increases the difficulty of mental rotation, it might not prevent the formation of associations between a given stimulus and the required response (i.e., stimulus-response associations). After washout, these latent stimulusresponse associations could have been reexpressed, eliciting faster subsequent when reach solutions at training matched that at test, and interference when the reach solutions differed. Intriguingly, participants reengaged a now-maladaptive reach solution, even when they were obviously able to immediately disengage it at washout under similar time pressure. Perhaps the learnt reach solution was triggered by some contextual cue (e.g., experiencing errors to the side of targets), and this prepotent response to the trigger was poorly inhibited under time pressure. This is consistent with the tendency to reexpress learnt stimulusresponse associations, even when they result in undesired outcomes (Dolan and Dayan, 2013). Alternatively, interference might result from prior training in mental rotation in the opposite direction (Sack et al., 2007). One clue to identify the more likely explanation comes from findings of deficient savings (Marinelli et al., 2009; Bédard and Sanes, 2011; Leow et al., 2012, 2013) and anterograde interference in Parkinson's disease (Leow et al., 2013). Parkinson's disease patients are better than controls when adapting to a rotation opposite to that previously experienced (Leow et al., 2013). This seems unlikely to result from superior capacity to disengage effects of previous mental rotation in an opposite direction. Deficient learning of stimulus-response associations in Parkinson's disease (Knowlton et al., 1996; Foerde, 2018) seems to be the more likely culprit. Thus, we interpret our data as evidence for the contribution of stimulus-response associations to sensorimotor memories.

The insight that stimulus-response associations contribute to sensorimotor memories has implications for work examining how the brain stores and retrieves sensorimotor memories. Associative learning relies on corticostriatal circuits (Toni et al., 2001; Yin and Knowlton, 2006; Rueda-Orozco and Robbe, 2015). During adaptation, learning stimulus-response associations might similarly rely on corticostriatal circuits, consistent with the abovementioned work in Parkinson's disease, and with imaging work implicating the basal ganglia in adaptation (Shadmehr and Holcomb, 1997, 1999; Diedrichsen et al., 2005; Seidler et al., 2006). In particular, target errors activate the striatum (Diedrich- sen et al., 2005) and area 7 of the parietal cortex (Diedrichsen et al., 2005; Inoue and Kitazawa, 2018). We speculate that task errors trigger an assessment of task state by the basal ganglia, prompting remapping of the relationship between goals and movement plans by parietal cortex area 7. Desirable task outcomes (e.g., reduced task error) might trigger the formation of stimulus-response associations, which is known to concurrently alter activity in the dorsal premotor cortex and parts of the putamen receiving inputs from the dorsal premotor cortex (Brasted and Wise, 2004). Thus, the characteristic changes in motor/dorsal premotor cortex preparatory activity seen in sensorimotor adaptation (e.g., Li et al., 2001; Paz et al., 2003; Mandelblat-Cerf et al., 2011; Perich et al., 2018; Zhou et al., 2019) might partly reflect how movement plans are affected by stimulus-response associations, as well as by implicit remapping in response to SPEs that occur via changes in functional connectivity between the cerebellum, area 5 of the parietal cortex, and motor cortex (Tanaka et al., 2009; Haar et al., 2015; Inoue and Kitazawa, 2018). Notably, our current data suggest that persistent sensorimotor memories that improve relearning rely primarily on corticostriatal circuits, rather than corticocerebellar circuits. Future work involving neural recordings that dissociate task and SPEs is required to test this prediction.

In conclusion, our results show that failures to attain movement goals, or task errors, are a fundamental driver of latent memories in sensorimotor adaptation. Sensorimotor memories are influenced not only by time-consuming, goal-directed processes (e.g., mental rotation), but also by rapidly accessible, stimulus-response associations. The data demonstrate the richness of behavioral responses to sensorimotor perturbations, and suggest that future efforts to characterize the neural mechanisms underpinning persistent sensorimotor memories should benefit from explicit dissociation of the distinct behavioral processes involved.

\section{References}

Abramson LY, Seligman ME, Teasdale JD (1978) Learned helplessness in humans: critique and reformulation. J Abnorm Psychol 87:49-74.

Anguera JA, Bernard JA, Jaeggi SM, Buschkuehl M, Benson BL, Jennett S, Humfleet J, Reuter-Lorenz PA, Jonides J, Seidler RD (2012) The effects of working memory resource depletion and training on sensorimotor adaptation. Behav Brain Res 228:107-115.

Avraham G, Keizman M, Shmuelof L (2020) Environmental consistency modulation of error sensitivity during motor adaptation is explicitly controlled. J Neurophysiol 123:57-69.

Bédard P, Sanes JN (2011) Basal ganglia-dependent processes in recalling learned visual-motor adaptations. Exp Brain Res 209:385-393.

Benson BL, Anguera JA, Seidler RD (2011) A spatial explicit strategy reduces error but interferes with sensorimotor adaptation. J Neurophysiol 105: 2843-2851.

Benson JS, Kennelly KJ (1976) Learned helplessness: the result of uncontrollable reinforcements or uncontrollable aversive stimuli? J Pers Soc Psychol 34:138.

Bhat RB, Sanes JN (1998) Cognitive channels computing action distance and direction. J Neurosci 18:7566-7580.

Bostan AC, Strick PL (2018) The basal ganglia and the cerebellum: nodes in an integrated network. Nat Rev Neurosci 19:338-350.

Brasted PJ, Wise SP (2004) Comparison of learning-related neuronal activity in the dorsal premotor cortex and striatum. Eur J Neurosci 19: 721-740.

Braun DA, Aertsen A, Wolpert DM, Mehring C (2009) Motor task variation induces structural learning. Curr Biol 19:352-357.

Crawford JD, Henriques DY, Medendorp WP (2011) Three-dimensional transformations for goal-directed action. Annu Rev Neurosci 34:309331.

Cunningham HA (1989) Aiming error under transformed spatial mappings suggests a structure for visual-motor maps. J Exp Psychol Hum Percept Perform 15:493-506. 
de Brouwer AJ, Albaghdadi M, Flanagan JR, Gallivan JP (2017) Gaze behaviour during sensorimotor adaptation parcellates the explicit and implicit contributions to learning. J Neurophysiol 120:1602-1615.

Diedrichsen J, Hashambhoy Y, Rane T, Shadmehr R (2005) Neural correlates of reach errors. J Neurosci 25:9919-9931.

Dietz V, Zijlstra W, Duysens J (1994) Human neuronal interlimb coordination during split-belt locomotion. Exp Brain Res 101:513-520.

Dolan RJ, Dayan P (2013) Goals and habits in the brain. Neuron $80: 312-325$

Ebbinghaus H (1964) Memory: A contribution to experimental psychology. Available at https://psycnet.apa.org/record/1965-11444-000.

Favilla M, De Cecco E (1996) Parallel direction and extent specification of planar reaching arm movements in humans. Neuropsychologia 34:609-613.

Fernandez-Ruiz J, Wong W, Armstrong IT, Flanagan JR (2011) Relation between reaction time and reach errors during visuomotor adaptation. Behav Brain Res 219:8-14.

Foerde K (2018) What are habits and do they depend on the striatum? A view from the study of neuropsychological populations. Curr Opin Behav Sci 20:17-24.

Galea JM, Sami SA, Albert NB, Miall RC (2010) Secondary tasks impair adaptation to step- and gradual-visual displacements. Exp Brain Res 202: 473-484.

Gallistel CR, Fairhurst S, Balsam P (2004) The learning curve: implications of a quantitative analysis. Proc Natl Acad Sci U S A 101:13124-13131.

Georgopoulos AP, Massey JT (1987) Cognitive spatial-motor processes: 1. The making of movements at various angles from a stimulus direction. Exp Brain Res 65:361-370.

Gonzalez Castro LN, Hadjiosif AM, Hemphill MA, Smith MA (2014) Environmental consistency determines the rate of motor adaptation. Curr Biol 24:1050-1061.

Haar S, Donchin O, Dinstein I (2015) Dissociating visual and motor directional selectivity using visuomotor adaptation. J Neurosci 35:6813-6821.

Hadjiosif A, Smith M (2013) Savings is restricted to the temporally labile component of motor adaptation. In: Translational and computational motor control. Washington, DC.

Haith AM, Huberdeau DM, Krakauer JW (2015) The influence of movement preparation time on the expression of visuomotor learning and savings. J Neurosci 35:5109-5117.

Heffley W, Song EY, Xu Z, Taylor BN, Hughes MA, McKinney A, Joshua M, Hull C (2018) Coordinated cerebellar climbing fiber activity signals learned sensorimotor predictions. Nat Neurosci 21:1431-1441.

Herzfeld DJ, Vaswani PA, Marko MK, Shadmehr R (2014) A memory of errors in sensorimotor learning. Science 345:1349-1353.

Howard IS, Ingram JN, Wolpert DM (2009) A modular planar robotic manipulandum with end-point torque control. J Neurosci Methods 181: $199-211$.

Huang VS, Haith A, Mazzoni P, Krakauer JW (2011) Rethinking motor learning and savings in adaptation paradigms: model-free memory for successful actions combines with internal models. Neuron 70:787-801.

Huberdeau DM, Haith AM, Krakauer JW (2015) Formation of a long-term memory for visuomotor adaptation following only a few trials of practice. J Neurophysiol 114:969-977.

Huberdeau DM, Krakauer JW, Haith AM (2019) Practice induces a qualitative change in the memory representation for visuomotor learning. J Neurophysiol 122:1050-1059.

Inoue $\mathrm{M}$, Kitazawa S (2018) Motor error in parietal area 5 and target error in area 7 drive distinctive adaptation in reaching. Curr Biol 28:22502262.e3.

Izawa J, Shadmehr R (2011) Learning from sensory and reward prediction errors during motor adaptation. PLoS Comput Biol 7:e1002012.

Kim HE, Parvin DE, Ivry RB (2019) The influence of task outcome on implicit motor learning. Elife 8:363606.

Knowlton BJ, Mangels JA, Squire LR (1996) A neostriatal habit learning system in humans. Science 273:1399-1402.

Koopman J, Howe M, Hollenbeck JR, Sin H-P (2015) Small sample mediation testing: Misplaced confidence in bootstrapped confidence intervals. Journal of Applied Psychology 100:194.

Kostadinov D, Beau M, Blanco-Pozo M, Häusser M (2019) Predictive and reactive reward signals conveyed by climbing fiber inputs to cerebellar Purkinje cells. Nat Neurosci 22:950-962.
Krakauer JW, Hadjiosif AM, Xu J, Wong AL, Haith AM (2019) Motor learning. Compr Physiol 9:613-663.

Landi SM, Baguear F, Della-Maggiore V (2011) One week of motor adaptation induces structural changes in primary motor cortex that predict long-term memory one year later. J Neurosci 31:11808-11813.

Leow LA, Loftus AM, Hammond GR (2012) Impaired savings despite intact initial learning of motor adaptation in Parkinson's disease. Exp Brain Res 218:295-304.

Leow LA, de Rugy A, Loftus AM, Hammond G (2013) Different mechanisms contributing to savings and anterograde interference are impaired in Parkinson's disease. Front Hum Neurosci 7:55.

Leow LA, de Rugy A, Marinovic W, Riek S, Carroll TJ (2016) Savings for visuomotor adaptation require prior history of error, not prior repetition of successful actions. J Neurophysiol 116:1603-1614.

Leow LA, Gunn R, Marinovic W, Carroll TJ (2017) Estimating the implicit component of visuomotor rotation learning by constraining movement preparation time. J Neurophysiol 118:666-676.

Leow LA, Marinovic W, de Rugy A, Carroll TJ (2018) Task errors contribute to implicit aftereffects in sensorimotor adaptation. Eur J Neurosci 48 : 3397-3409.

Li CS, Padoa-Schioppa C, Bizzi E (2001) Neuronal correlates of motor performance and motor learning in the primary motor cortex of monkeys adapting to an external force field. Neuron 30:593-607.

Maeda RS, McGee SE, Marigold DS (2018) Long-term retention and reconsolidation of a visuomotor memory. Neurobiol Learn Mem 155:313-321.

Malone LA, Bastian AJ (2010) Thinking about walking: effects of conscious correction versus distraction on locomotor adaptation. J Neurophysiol 103:1954-1962.

Mandelblat-CerfY, Novick I, Paz R, Link Y, Freeman S, Vaadia E (2011) The neuronal basis of long-term sensorimotor learning. J Neurosci 31: 300-313.

Marinelli L, Crupi D, Di Rocco A, Bove M, Eidelberg D, Abbruzzese G, Ghilardi MF (2009) Learning and consolidation of visuo-motor adaptation in Parkinson's disease. Parkinsonism Relat Disord 15:6-11.

Mazzoni P, Krakauer JW (2006) An implicit plan overrides an explicit strategy during visuomotor adaptation. J Neurosci 26:3642-3645.

McDougle SD, Taylor JA (2019) Dissociable cognitive strategies for sensorimotor learning. Nat Commun 10:40.

Morehead JR, Qasim SE, Crossley MJ, Ivry R (2015) Savings upon re-aiming in visuomotor adaptation. J Neurosci 35:14386-14396.

Orban de Xivry JJ, Lefèvre P (2015) Formation of model-free motor memories during motor adaptation depends on perturbation schedule. J Neurophysiol 113:2733-2741

Paz R, Boraud T, Natan C, Bergman H, Vaadia E (2003) Preparatory activity in motor cortex reflects learning of local visuomotor skills. Nat Neurosci 6:882-890.

Pellizzer G, Georgopoulos AP (1993) Common processing constraints for visuomotor and visual mental rotations. Exp Brain Res 93:165-172.

Perich MG, Gallego JA, Miller LE (2018) A neural population mechanism for rapid learning. Neuron 100:964-976.e7.

Pidoux L, Le Blanc P, Levenes C, Leblois A (2018) A subcortical circuit linking the cerebellum to the basal ganglia engaged in vocal learning. Elife 7:e32167.

Preacher KJ, Hayes AF (2004) SPSS and SAS procedures for estimating indirect effects in simple mediation models. Behav Res Methods Instrum Comput 36:717-731.

Roth S (1980) A revised model of learned helplessness in humans. J Pers 48:103-133

Rueda-Orozco PE, Robbe D (2015) The striatum multiplexes contextual and kinematic information to constrain motor habits execution. Nat Neurosci 18:453-460.

Sack AT, Lindner M, Linden DE (2007) Object- and direction-specific interference between manual and mental rotation. Percept Psychophys 69: $1435-1449$.

Savoie FA, Thénault F, Whittingstall K, Bernier PM (2018) Visuomotor prediction errors modulate EEG activity over parietal cortex. Sci Rep $8: 12513$

Schouten JF, Bekker JA (1967) Reaction time and accuracy. Acta Psychol (Amst) 27:143-153

Schween R, Taube W, Gollhofer A, Leukel C (2014) Online and post-trial feedback differentially affect implicit adaptation to a visuomotor rotation. Exp Brain Res 232:3007-3013. 
Seidler RD, Noll DC, Chintalapati P (2006) Bilateral basal ganglia activation associated with sensorimotor adaptation. Exp Brain Res 175:544-555.

Shadmehr R, Holcomb HH (1997) Neural correlates of motor memory consolidation. Science 277:821-825.

Shadmehr R, Holcomb HH (1999) Inhibitory control of competing motor memories. Exp Brain Res 126:235-251.

Shadmehr R, Mussa-Ivaldi FA (1994) Adaptive representation of dynamics during learning of a motor task. J Neurosci 14:3208-3224.

Shrout PE, Bolger N (2002) Mediation in experimental and nonexperimental studies: new procedures and recommendations. Psychol Methods $7: 422-445$.

Tanaka H, Sejnowski TJ, Krakauer JW (2009) Adaptation to visuomotor rotation through interaction between posterior parietal and motor cortical areas. J Neurophysiol 102:2921-2932.

Taylor JA, Ivry RB (2011) Flexible cognitive strategies during motor learning. PLoS Comput Biol 7:e1001096.

Taylor JA, Ivry RB (2014) Cerebellar and prefrontal cortex contributions to adaptation, strategies, and reinforcement learning. Prog Brain Res 210:217-253.

Taylor JA, Thoroughman KA (2007) Divided attention impairs human motor adaptation but not feedback control. J Neurophysiol 98:317-326.

Taylor JA, Thoroughman KA (2008) Motor adaptation scaled by the difficulty of a secondary cognitive task. PLoS One 3:e2485.
Toni I, Ramnani N, Josephs O, Ashburner J, Passingham RE (2001) Learning arbitrary visuomotor associations: temporal dynamic of brain activity. Neuroimage 14:1048-1057.

Vickers AJ (2001) The use of percentage change from baseline as an outcome in a controlled trial is statistically inefficient: a simulation study. BMC Med Res Methodol 1:6.

Vickers AJ (2014) Change/percent change from baseline. In: Wiley encyclopedia of clinical trials (D'Agostino RB, Sullivan L, Massaro J, eds), pp 1-7. New York: Wiley.

von Helmholtz H (1924) Helmholtz's treatise on physiological optics, Vol 1, Transactions. Rochester, NY: Optical Society of America.

Yin HH, Knowlton BJ (2006) The role of the basal ganglia in habit formation. Nat Rev Neurosci 7:464-476.

Zach N, Kanarek N, Inbar D, Grinvald Y, Milestein T, Vaadia E (2005) Segregation between acquisition and long-term memory in sensorimotor learning. Eur J Neurosci 22:2357-2362.

Zarahn E, Weston GD, Liang J, Mazzoni P, Krakauer JW (2008) Explaining savings for visuomotor adaptation: linear time-invariant state-space models are not sufficient. J Neurophysiol 100:2537-2548.

Zhou X, Tien RN, Ravikumar S, Chase SM (2019) Distinct types of neural reorganization during long-term learning. J Neurophysiol 121:1329_ 1341. 